\title{
Typ-2-Diabetes - Kriterien für die Wahl des Antidiabetikums
}

Baptist Gallwitz

\begin{abstract}
Die moderne Behandlung des Typ-2-Diabetes ist patientenorientiert: Der HbA 1 -Zielwert wird individuell festgelegt - abhängig von Alter, Krankheitsdauer und Komorbiditäten des Betroffenen. Zur stufenweisen Therapie stehen mittlerweile mehrere Medikamente mit unterschiedlichen Wirkstoffen zur Verfügung. Eine leitliniengerechte, evidenzbasierte Einordnung der möglichen Therapien soll als Orientierung im Praxisalltag dienen.
\end{abstract}

Patientenorientiert vorgehen I Die Patientenpopulation bei Typ-2-Diabetes ist sehr heterogen. Deshalb scheint eine Festlegung des $\mathrm{HbA}_{1 \mathrm{c}}$-Zielkorridors auf der Basis individueller, patientenbezogener Kriterien zielführend $\mathrm{zu}$ sein. Für solch ein patientenorientiertes Vorgehen haben sich auch die American Diabetes Association (ADA) und die European Association for the Study of Diabetes (EASD) eingesetzt und 2012 ein Konsensus-Statement veröffentlicht. Dieses wurde 2015 aktualisiert und weiterentwickelt [1].

Die Nationale Versorgungsleitlinie hat den Grundsatz einer patientenorientierten Festlegung des $\mathrm{HbA}_{1 \mathrm{c}}$-Zielwerts in ihre aktuelle Version aufgenommen.

Ferner fokussiert sie auch darauf, Hypoglykämien und eine therapiebedingte Gewichtszunahme zu vermeiden [2].

\section{Individuelle Behandlungsziele}

Nicht zu streng einstellen | Ziel einer Diabetesbehandlung ist die langfristige glykämische Kontrolle. Durch Senkung des $\mathrm{HbA}_{1 \mathrm{c}}$-Werts lässt sich das Risiko für Hyperglykämie-bedingte Komplikationen (sowohl mikro- als auch makrovaskulärer Art) reduzieren. Allerdings wurden mögliche Nachteile eines allzu intensiven, glucozentrischen Therapieansatzes durch die Ergebnisse der ACCORD-, ADVANCE- und VADT-Studien deutlich: Häufig waren hypoglykämische Episoden die Folge [3]. Die Ergebnisse dieser Studien haben die Therapieziele bei Typ-2-Diabetes differenzierter werden lassen.

Individuelle Therapie erfolgsentscheidend | Typ2-Diabetes ist eine multifaktorielle Erkrankung und häufig mit unterschiedlichen Komorbiditäten assoziiert. Dies bedeutet, dass Betroffene nicht in gleicher Weise von einem straff vorgegebenen Behandlungskonzept profitieren und der jeweilige Patient im Fokus stehen sollte.
So ist die individuelle Therapiegestaltung erfolgsentscheidend und wirkt sich auch auf die Therapieadhärenz des Patienten aus.

Blutzucker-Zielwert | Die Nationale Versorgungsleitlinie empfiehlt zur Primärprävention von Folgekomplikationen einen $\mathrm{HbA}_{1 \mathrm{c}}$-Zielkorridor von 6,5-7,5\% [2]. Um den Blutzucker-Zielwert innerhalb dieses Korridors festzulegen, sollten individuelle Aspekte, wie z.B. die Diabetesdauer, berücksichtigt werden ( $\triangleright$ Tab. 1) [1, 2]. Nach individueller Situation des Patienten können auch Zielwerte außerhalb dieses Korridors sinnvoll sein.

\section{Lebensstilintervention und Monotherapie mit Metformin}

Änderung der Lebensweise I Der Blutzuckerwert sollte möglichst früh im Krankheitsverlauf eingestellt werden: Die Zeitspanne, die bis zur individuell idealen glykämischen Kontrolle vergeht, kann einen Einfluss auf das sogenannte metabolische Gedächtnis haben. In erster Linie kommt es darauf an, durch Schulung des Patienten eine wirksame Lebensstilintervention bezüglich der Ernährung und der körperlichen Aktivität zu erreichen.

Metformin-Monotherapie I Wurde der individuell festgelegte $\mathrm{HbA}_{1 \mathrm{c}}$-Zielwert trotz der Lebensstilintervention innerhalb von 3-6 Monaten nicht erreicht, ist Metformin das präferierte Antidiabetikum in der ersten pharmakologischen Therapiestufe ( Tab. 2) [1, 2]. Als Monotherapie bewirkt Metformin eine $\mathrm{HbA}_{1 \mathrm{c}}$-Reduktion von $1-2 \%$. Ferner erniedrigt es das LDL-Cholesterin und die Triglycerid-Konzentrationen [4]. Die Bewertung neuer Daten zeigt, dass Metformin in reduzierter Dosis nun auch bei Patienten mit Niereninsuffizienz bis zu einer Kreatinin-Clearance von $45 \mathrm{ml} / \mathrm{min}$ eingesetzt werden kann [5]. Wird unter Metformin-Monotherapie innerhalb von etwa 3-6 Monaten der individuell festgelegte $\mathrm{HbA}_{1 \mathrm{c}}$-Zielwert nicht erreicht, sollte ein weiteres Antidiabetikum hinzugenommen werden [1, 2]. 
Faktoren

$$
\begin{aligned}
& \text { Risiken in Zusammenhang mit } \\
& \text { Hypoglykämien und anderen } \\
& \text { unerwünschten Wirkungen }
\end{aligned}
$$$$
\text { bekannte Diabetesdauer }
$$$$
\text { Lebenserwartung }
$$$$
\text { entscheidende Komorbiditäten }
$$$$
\text { manifeste Gefäßkomplikationen }
$$$$
\text { Patienteneinstellung und }
$$$$
\text { Therapieerwartung }
$$

\section{Ressourcen und Unterstützung}

Modifizierbar?

streng (<7\%)

nein

niedrig

nein

nein

nein

nein

potenziell

(1)

potenziell

lang

fehlend

fehlend
$\mathrm{HbA}_{1 \mathrm{c}}$-Einstellung

weniger streng (>7\%)

hoch

neu diagnostiziert

hohe Motivation / Adhärenz; exzellente Selbstbehandlungskompetenz

leicht verfügbar
Tab. 1 Ansatz zum

Hyperglykämie-Management [1]. Teils modifizierbare, teils nicht modifizierbare Faktoren entscheiden darüber, ob das $\mathrm{HbA}_{1 \mathrm{c}}$ eher streng oder eher weniger streng eingestellt werden sollte. Die Übergänge sind fließend.
Alternativen | Ein alternativer pharmakologischer Ansatz zu einer Metformin-Monotherapie ist notwendig, wenn eine Metformin-Unverträglichkeit oder eine Kontraindikation vorliegt (z.B. schwere chronische Erkrankungen mit ausgeprägter Hypoxieneigung oder eine Alkoholkrankheit). Das Antidiabetikum sollte man unter Berücksichtigung der jeweiligen Wirkstoffeigenschaften und der individuellen patientenspezifischen Faktoren wählen.

Da es jedoch gegenwärtig an langfristigen vergleichenden Studien mit klinisch relevanten Endpunkten zur Wirksamkeit der einzelnen Antidiabetika mangelt, können keine einheitlichen Empfehlungen zur Wahl des geeignetsten Wirkstoffes ausgesprochen werden.

Im Folgenden werden daher die zur Verfügung stehenden Optionen anhand wichtiger Behandlungskriterien eingeordnet. Auf eine Erörterung von Pioglitazon und der $\alpha$-GlucosidaseInhibitoren wird hinsichtlich ihrer geringen Bedeutung in der Therapielandschaft in diesem $\mathrm{Zu}$ sammenhang verzichtet.

\section{Glukoseunabhängige insulinotrope Substanzen}

Sulfonylharnstoffe und Glinide I Diese Wirkstoffe stimulieren die Insulinsekretion glukoseunabhängig. In Placebo-kontrollierten Studien bewirkten Sulfonylharnstoffe eine durchschnittliche $\mathrm{HbA}_{1 \mathrm{c}}-$ Reduktion von -0,8\% [6]. Die Wirkung der Glinide war mit 0,7\% etwas geringer [6]. Unterschiede zwischen beiden Substanzklassen zeigen sich auch in der Pharmakokinetik. So ist die Halbwertszeit der Glinide kürzer als die der Sulfonylharnstoffe, weshalb Glinide öfter am Tag mahlzeitenbezogen eingenommen werden müssen.
Da Sulfonylharnstoffe und Glinide glukoseunabhängig wirken, d. h. ohne physiologische Limitation, sind sie mit einem vergleichsweise hohen Hypoglykämierisiko assoziiert (durchschnittliches relatives Risiko in Placebokontrollierten Studien: 2,63 bzw. 7,92) [6].

Gewichtszunahme | Die Ergebnisse Placebokontrollierter, klinischer Studien haben zudem gezeigt, dass der Einsatz beider Substanzen häufig mit einer Zunahme des Körpergewichts verbunden ist (2,0 kg unter Sulfonylharnstoffen bzw. $0,9 \mathrm{~kg}$ unter Gliniden) [6]. In der UK Prospective Diabetes Study (UKPDS) 33 wurde unter Sulfonylharnstoffen im Vergleich zu einer konventionellen Therapie (Ernährungsumstellung) Präparatabhängig eine signifikante durchschnittliche Zunahme des Körpergewichts um bis zu 2,6 kg beobachtet [7]. Der Einfluss von Sulfonylharnstoffen und Gliniden auf kardiovaskuläre Parameter ist bislang nicht eindeutig geklärt.

\section{Glukoseabhängige insulinotrope Substanzen}

Inkretinbasierte Medikamente I Glucagon-LikePeptide-1-Rezeptor-Agonisten (GLP-1-RA) und Dipeptidyl-Peptidase (DPP)-4-Inhibitoren bilden die Gruppe der inkretinbasierten Medikamente, deren insulinotrope Wirkung vom Blutzuckerspiegel abhängig ist. Placebo-kontrollierte, klinische Studien haben gezeigt, dass die $\mathrm{HbA}_{1 \mathrm{c}}-\mathrm{Re}-$ duktion unter GLP-1-RA durchschnittlich $-1,0 \%$, und unter DPP-4-Inhibitoren durchschnittlich $-0,8 \%$ beträgt [6]. GLP-1-RA und DPP-4-Hemmer sind mit einem vergleichsweise geringen Hypoglykämierisiko assoziiert (relatives Risiko gegenüber Placebo: 0,94 bzw. 0,67). Durch eine praktisch altersunabhängige Pharmakokinetik und Verträglichkeit erzielen GLP-1-RA wie Liraglutid 
auch im Risikokollektiv der älteren Diabetespatienten eine effektive glykämische Kontrolle [8].

GLP-1-RA: Reduktion des Körpergewichts | Sowohl das natürliche GLP-1 als auch GLP-1-RA besitzen zusätzlich extrapankreatische Effekte:

- So zeigte eine direkte 26-wöchige Vergleichsstudie, dass die GLP-1-RA Exenatide (RetardForm) und Liraglutid eine dosisabhängige Reduktion des Körpergewichts um bis zu $2,7 \mathrm{~kg}$ bzw. 3,6 kg bewirkten ( $\mathrm{p}=0,0005)$ [9].

- Unter Dulaglutid wurde im gleichen Zeitraum eine Reduktion des Körpergewichts um 2,9 kg beobachtet (im Vergleich zu 3,6 kg unter Liraglutid; $p=0,011$ ) [10].

- Auch Lixisenatid und Albiglutid erwiesen sich im Rahmen direkter Vergleichsstudien mit Liraglutid als gewichtsreduzierend $(-1,6 \mathrm{~kg}$ nach 28 Tagen bzw. $-0,6 \mathrm{~kg}$ nach 32 Wochen) [11, 12].

Allerdings war dieser Effekt unter dem Komparator Liraglutid jeweils signifikant stärker $(-2,4 \mathrm{~kg}$, $\mathrm{p}<0,01$ bzw. $-2,2 \mathrm{~kg}, \mathrm{p}<0,0001)[11,12]$.

DPP-4-Inhibitoren: gewichtsneutral | Für die DPP-4-Inhibitoren konnten vergleichbare Effekte bisher nicht beobachtet werden, weshalb sie allgemein als gewichtsneutral angesehen werden. In Placebo-kontrollierten Studien wurde eine durchschnittliche Gewichtsreduktion von $0,1 \mathrm{~kg}$ bzw. $1,8 \mathrm{~kg}$ für DPP-4-Hemmer bzw. GLP-1-RA beobachtet [6].

Wirkung auf kardiovaskuläre Risikofaktoren I GLP-1-RA haben einen günstigen Effekt auf kardiovaskuläre Risikofaktoren - wie den systolischen Blutdruck und das Lipidprofil [13]. DPP4-Inhibitoren stellten sich als neutral im Hinblick auf einen primären gemischten kardiovaskulären Endpunkt im Vergleich zur Standardtherapie bzw. Placebo heraus $[14,15]$.

\section{Insulinunabhängig wirkende Medikamente}

Geringes Risiko für Hypoglykämien | Substanzen, die die physiologische Aktivität des renal exprimierten Sodium/Glukose-Co-Transporters
(SGLT)-2 hemmen, verfolgen einen insulinunabhängigen Wirkansatz. In der Monotherapie bewirken sie eine Reduktion des $\mathrm{HbA}_{1 \mathrm{c}}$-Werts um $-0,3$ bis $-1,0 \%[16]$.

Aufgrund ihres Wirkmechanismus ist das Risiko wirkstoffinduzierter Hypoglykämien sehr gering $(0,9 \%$ und $4,3 \%)$ [16].

Die durch SGLT-2-Inhibitoren hervorgerufene vermehrte Glukose-Exkretion war in klinischen Studien zudem mit einer Reduktion des Körpergewichts um bis zu $3 \mathrm{~kg}$ assoziiert [17]. Eine Zunahme hypoglykämischer Ereignisse wurde vor allem bei Kombinationstherapien mit Sulfonylharnstoffen beobachtet [16].

Ketoazidosen I Die Ergebnisse einer kürzlich vorgestellten Endpunktstudie zu Empagliflozin zeigten unter anderem eine reduzierte kardiovaskuläre Sterblichkeit bei Patienten mit entsprechender Vorbelastung [23]. Ein Informationsbrief des Bundesinstituts für Arzneimittel und Medizinprodukte berichtet über Ketoazidosen, die unter einer Therapie mit SGLT-2-Inhibitoren auftreten. Er verweist insbesondere auf die fehlende Zulassung dieser Substanzgruppe zur Behandlung von Menschen mit Typ-1-Diabetes [18].

\section{Insulin}

Effektive $\mathrm{HbA}_{1 \mathrm{c}}$-Reduktion I Insulin und Insulinanaloga bilden die Wirkstoffklasse mit der größten klinischen Erfahrung zur Therapie des Typ2-Diabetes. Sie ist im Vergleich zu anderen Antidiabetika besonders effektiv in Bezug auf die $\mathrm{HbA}_{1 \mathrm{c}}$-Reduktion und ist häufig notwendig, um langfristig eine normoglykämische Einstellung der Patienten zu erreichen. Es wurde eine $\mathrm{HbA}_{1 c^{-}}$ Reduktion von bis zu 5\% (in Kombination mit Metformin) beobachtet [19]. Allerdings ist das Risiko hypoglykämischer Ereignisse unter Insulingabe erhöht, kann aber durch den Einsatz kurzwirksamer und/oder langwirksamer Insulinanaloga vermindert werden.

\begin{tabular}{llll} 
Wirkstoff & $\begin{array}{l}\text { Wirksamkeit } \\
\left(\mathbf{H b A}_{1 \mathbf{c}} \text {-Reduktion) }\right.\end{array}$ & Hypoglykämierisiko & $\begin{array}{l}\text { Auswirkung auf das } \\
\text { Körpergewicht }\end{array}$ \\
\hline Metformin & ++ & niedrig & neutral \\
Sulfonylharnstoff & ++ & hoch & $\uparrow$ \\
Glinide & + & moderat & $\uparrow$ \\
GLP-1-RA & ++ & niedrig & $\downarrow \downarrow$ \\
DPP-4-Hemmer & + & niedrig & neutral \\
Q-Glucosidase-Hemmer & + & niedrig & $\downarrow \downarrow$ \\
SGLT2-Inhibitoren & + & niedrig & $\downarrow \downarrow$
\end{tabular}

Tab. 2 Wirkstoffeigenschaften im indirekten Vergleich. Nur eingeschränkte Vergleichbarkeit auf Basis der vorhandenen Daten $[1,4,6$, $16,17]$. 


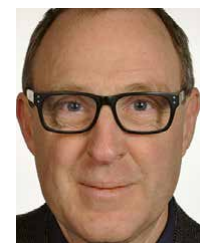

Prof. Dr. med.

Baptist Gallwitz

ist Stellv. Direktor der

Medizinischen Klinik IV,

Universitätsklinikum

Tübingen.

baptist.gallwitz@med. uni-tuebingen.de

\section{Interessenkonflikt}

Der Autor gibt an, Vortragstätigkeiten für AstraZeneca, Boehringer Ingelheim, Bristol Myers Squibb, Berlin-Chemie, Novartis, Novo Nordisk, MSD, Lilly, Roche und Sanofi durchgeführt zu haben. Er war Mitglied in Advisory Boards bei AstraZeneca, Boehringer Ingelheim, MSD, Novo Nordisk, Lilly und Roche.

DOI 10.1055/s-0041-106694 Dtsch Med Wochenschr 2016; 141: 285-288

(c) Georg Thieme Verlag KG . Stuttgart · New York .

ISSN 0012-0472
Gewichtszunahme | Ein weiterer Aspekt in der Insulintherapie ist die zum Teil deutliche Körpergewichtszunahme der Patienten. Diese ist vermutlich insbesondere einer verminderten Glukosurie zuzuschreiben.

\section{Divergenzen in den Leitlinien zur Behandlung des Typ-2-Diabetes}

Uneinigkeit bei den Gesellschaften I In die aktuelle deutsche Nationale Versorgungsleitlinie wurde der individuelle Ansatz der Diabetestherapie aufgenommen, wenngleich zwischen den verantwortenden Organisationen (DDG, DGIM, DEGAM, AkdÄ) noch Divergenzen bestehen - vor allem bei der Grundlage der Therapieentscheidung [2]:

- DEGAM und AkdÄ verfolgen einen Therapiealgorithmus, basierend auf einem eher konservativen Evidenzbegriff. So werden primär nur Wirkstoffe und Wirkstoffkombinationen empfohlen, für die eine entsprechende Evidenz aus Endpunktstudien gegeben ist.

- Demgegenüber befürworten DDG und DGIM einen flexibleren Einsatz von Antidiabetika, unter Berücksichtigung vor allem des Hypoglykämiepotenzials [22]. Vor- und Nachteile sind aus ihrer Sicht abhängig von der individuellen Situation des Patienten einzuordnen.

Verschiedene Leitlinienempfehlungen I Entsprechend dieser Divergenzen ergeben sich - abgesehen von einer Pharmako-Monotherapie mit Metformin als erste Wahl - unterschiedliche Leitlinienempfehlungen.

- DEGAM und AkdÄ bevorzugen eine Kombinationstherapie aus Metformin/Humaninsulin oder Metformin/Sulfonylharnstoff, da sie für diese Kombinationen einen Nutzennachweis sehen.

- DDG und DGIM erachten als wichtige Parameter einer zielführenden Diabetestherapie, das Auftreten hypoglykämischer Episoden und einer Zunahme des Körpergewichts zu verhindern [2]. Daher haben Sulfonylharnstoffe und Insulin hier einen geringeren Stellenwert.

Empfehlungen zu Dreifachkombinationen | Die Divergenz in den Leitlinien zeigt sich auch mit Blick auf eine mögliche Kombination von drei unterschiedlichen Antidiabetika plus Basistherapie, wenn unter einer Zweifachkombination das gewünschte $\mathrm{HbA}_{1 \mathrm{c}}$-Ziel nicht erreicht werden kann. Aus Sicht der DDG und DGIM liegen Studienergebnisse vor, die eine verbesserte glykämische Kontrolle unter Dreifachkombination andeuten $[20,21]$. Sie betonen jedoch, dass aufgrund der schwachen Datenlage eine engmaschige Überwachung des Patienten notwendig ist. Außerdem sollten nur jene Wirkstoffe kombiniert werden, deren Wirkmechanismen sich ergänzen. DEGAM und AkdÄ sehen in den vorliegenden Daten keine ausreichende Evidenz für dieses Vorgehen und raten daher grundsätzlich von einer Dreifachkombination antidiabetischer Wirkstoffe ab und favorisieren damit einen sehr viel früheren Einsatz von Insulin.

Konsequenz für Klinik und Praxis

- Die individuelle Situation des Patienten hat einen wesentlichen Einfluss auf die Festlegung des $\mathrm{HbA}_{1 c}$-Zielwerts. Dabei ist das allgemeine Ziel, die mit Hyperglykämien assoziierten Risikofaktoren zu mindern und Komplikationen zu vermeiden, um so die Morbidität und Mortalität zu reduzieren.

- Eine frühe Therapieoptimierung ist entscheidend für einen nachhaltigen Behandlungserfolg.

- Eine Lebensstil-intervenierende Basistherapie und eine Monotherapie mit Metformin bilden nach wie vor die Grundpfeiler im Therapiemanagement des Typ-2-Diabetes.

- Für die Kombinationstherapie stehen mittlerweile viele verschiedene Wirkstoffe zur Verfügung. Wichtige Aspekte für die Wahl eines geeigneten Antidiabetikums umfassen seine Wirksamkeit, die Risiken für Hypoglykämien und kardiovaskuläre Ereignisse sowie die Auswirkung auf das Körpergewicht.

- Eindeutige Empfehlungen können gegenwärtig nicht ausgesprochen werden, da Langzeitstudien mit harten Endpunkten noch fehlen - mit Ausnahme für den SGLT2-Hemmer Empagliflozin in einer Studie. Substanzen, wie die GLP-1-RA, zeigen erste vielversprechende Ergebnisse für eine verbesserte Therapie, wohingegen sich ungünstige Daten zu Sulfonylharnstoffen gemehrt haben.

\section{Danksagung}

Der Autor dankt Dr. Bastian Schmidt (Medical Writing Services) und der Firma Novo Nordisk für die Unterstützung bei der Erstellung des Manuskriptes.

\section{Literatur}

1 Inzucchi SE, Bergenstal RM, Buse JB et al. Management of hyperglycaemia in type 2 diabetes, 2015: a patient-centred approach. Update to a Position Statement of the American Diabetes Association and the European Association for the Study of Diabetes. Diabetologia 2015; 58: 429-442

2 Bundesärztekammer (BÄK), Kassenärztliche Bundesvereinigung (KBV), and Arbeitsgemeinschaft der Wissenschaftlichen Medizinischen Fachgesellschaften (AWMF): Nationale VersorgungsLeitlinie Therapie des Typ-2-Diabetes - Langfassung, 1. Auflage. Version 4. 2013. DOI: 10.6101/AZQ/000213

Vollständiges Literaturverzeichnis unter http://dx.doi.org/10.1055/s-0041-106694 
3 Terry T, Raravikar K, Chokrungvaranon N, Reaven PD. Does aggressive glycemic control benefit macrovascular and microvascular disease in type 2 diabetes? Insights from ACCORD, ADVANCE, and VADT. Curr Cardiol Rep 2012; 14: 79-88

4 Rodbard HW, Blonde L, Braithwaite SS et al. American Association of Clinical Endocrinologists medical guidelines for clinical practice for the management of diabetes mellitus. Endocr Pract 2007; 13 Suppl 1: 1-68.

5 Bundesinstitut für Arzneimittel und Medizinprodukte. Metformin: Aktualisierung der Fach- und Gebrauchsinformation hinsichtlich der Kontraindikation bei Patienten mit eingeschränkter Nierenfunktion. 2015. Im Internet: www.bfarm.de/ SharedDocs/Risikoinformationen/Pharmakovigilanz/DE/RI/2015/RI-metformin.html; letzter Zugriff: 2.12.2015

6 Phung OJ, Scholle JM, Talwar M, Coleman Cl. Effect of noninsulin antidiabetic drugs added to metformin therapy on glycemic control, weight gain, and hypoglycemia in type 2 diabetes. JAMA, 2010; 303: 1410-1418

7 UK Prospective Diabetes Study (UKPDS) Group. Intensive blood-glucose control with sulphonylureas or insulin compared with conventional treatment and risk of complications in patients with type 2 diabetes (UKPDS 33). Lancet 1998; 352: 837-853

8 Bode BW, Brett J, Falahati A, Pratley RE. Comparison of the efficacy and tolerability profile of liraglutide, a once-daily human GLP-1 analog, in patients with type 2 diabetes $\geq 65$ and $<65$ years of age: a pooled analysis from phase III studies. Am J Geriatr Pharmacother 2011. 9: 423-433

9 Buse JB, Nauck M, Forst T et al. Exenatide once weekly versus liraglutide once daily in patients with type 2 diabetes (DURATION-6): a randomised, open-label study. Lancet 2013; 381: 117-124

10 Dungan KM, Povedano ST, Forst T et al. Onceweekly dulaglutide versus once-daily liraglutide in metformin-treated patients with type 2 diabetes (AWARD-6): a randomised, open-label, phase 3, non-inferiority trial. Lancet 2014. 384: 1349-1357

11 Kapitza C, Forst T, Coester HV et al. Pharmacodynamic characteristics of lixisenatide once daily versus liraglutide once daily in patients with type 2 diabetes insufficiently controlled on metformin. Diabetes Obes Metab, 2013. 15: 642-649

12 Pratley RE, Nauck MA, Barnett AH et al. Onceweekly albiglutide versus once-daily liraglutide in patients with type 2 diabetes inadequately controlled on oral drugs (HARMONY 7): a randomised, open-label, multicentre, noninferiority phase 3 study. Lancet Diabetes Endocrinol 2014; 2: 289-297.
13 Mundil D, Cameron-Vendrig A, Husain M. GLP-1 receptor agonists: a clinical perspective on cardiovascular effects. Diab Vasc Dis Res 2012; 9 : 95-108

14 Green JB, Bethel MA, Armstrong PW. Effect of Sitagliptin on Cardiovascular Outcomes in Type 2 Diabetes. N Engl J Med 2015; 373: 232-242

15 Scirica BM, Bhatt DL, Braunwald E et al. Saxagliptin and cardiovascular outcomes in patients with type 2 diabetes mellitus. N Engl J Med 2013; 369: 1317-1326

16 Rosenwasser RF, Sultan S, Sutton D et al. SGLT-2 inhibitors and their potential in the treatment of diabetes. Diabetes Metab Syndr Obes 2013; 6 : 453-467

17 Riser Taylor S, Harris KB. The clinical efficacy and safety of sodium glucose cotransporter- 2 inhibitors in adults with type 2 diabetes mellitus. Pharmacotherapy 2013; 33: 984-999

18 Bundesinstitut für Arzneimittel und Medizinprodukte. Informationsbrief zu SGLT2-Inhibitoren: Risiko einer diabetischen Ketoazidose. Im Internet: www.bfarm.de/SharedDocs/Risikoinformationen/ Pharmakovigilanz/DE/RHB/2015/info-sglt2.html. 2015; letzter Zugriff: 2.12.2015

19 Lingvay I, Kaloyanova PF, Adams-Huet B et al. Insulin as initial therapy in type 2 diabetes: effective, safe, and well accepted. J Investig Med, 2007; 55: 62-68

20 Kendall DM, , Riddle MC, Rosenstock J. Effects of exenatide (exendin-4) on glycemic control over 30 weeks in patients with type 2 diabetes treated with metformin and a sulfonylurea. Diabetes Care 2005; 28: 1083-1091

21 Zinman B, Gerich J, Buse JB et al. Efficacy and safety of the human glucagon-like peptide- 1 analog liraglutide in combination with metformin and thiazolidinedione in patients with type 2 diabetes (LEAD-4 Met+TZD). Diabetes Care 2009; 32: $1224-1230$

22 Landgraf R, Kellerer M, Fach E et al. Praxisempfehlungen DDG/ DGIM. Diabetologie 2015; 10 (Suppl 2): $140-151$

23 Zinman B, Wanner C, Lachin JM et al. Empagliflozin, cardiovascular outcomes, and mortality in type 2 diabetes. N Engl ] Med 2015; 373: 2117-2128 